\title{
A novel compound heterozygous mutation in TTC8 identified in a Japanese patient
}

\author{
Shigeru Sato', Takeshi Morimoto ${ }^{1,2}$, Kikuko Hotta ${ }^{3}$, Takashi Fujikado ${ }^{1,2}$ and Kohji Nishida ${ }^{1}$
}

\begin{abstract}
Bardet-Biedl syndrome (BBS), characterized by rod-cone dystrophy, postaxial polydactyly, central obesity, hypogonadism, renal abnormalities, and mental retardation, is a rare autosomal recessive disorder. To date, 21 causative genes have been reported. Here we describe a Japanese BBS patient with a novel compound heterozygous mutation in TTC8. To the best of our knowledge, this is the first description of a BBS patient with a mutation in the TTC8 gene in Japan.
\end{abstract}

Bardet-Biedl syndrome (BBS) is a rare autosomal recessive disorder characterized by rod-cone dystrophy, postaxial polydactyly, central obesity, hypogonadism, renal abnormalities, and mental retardation. BBS is often complicated by strabismus/cataracts/astigmatism, diabetes mellitus, Hirschsprung disease, heart disease, and/or liver fibrosis. To date, 21 causative genes have been reported, comprising $\sim 80 \%$ of BBS genetic abnormalities $^{1,2}$. The remaining $20 \%$ of genetic abnormalities among BBS patients are not yet known. In the present study, we performed whole-exome sequencing (WES) of a classical BBS patient.

The patient was diagnosed with BBS at 8 years of age, in accordance with criteria reported previously ${ }^{3}$. Primary and secondary signs of BBS in this patient are listed in Table 1. When the patient first visited Osaka University Hospital at 17 years of age, his best-corrected visual acuity (BCVA) was 0.07 in the right eye and 0.2 in the left eye. At 28 years of age, his BCVA was 0.01 in the right eye and 0.04 in the left eye; he exhibited bilateral diffuse retinal degeneration, including macular atrophy, attenuated retinal vessels, and optic nerve head pallor with little pigmentary dispersion. His parents were not consanguineous. His mother showed

\footnotetext{
Correspondence: Shigeru Sato (s.sato@ophthal.med.osaka-u.ac.jp)

${ }^{1}$ Department of Ophthalmology, Osaka University Graduate School of Medicine, Osaka, Japan

${ }^{2}$ Department of Applied Visual Science, Osaka University Graduate School of Medicine, Osaka, Japan
}

Full list of author information is available at the end of the article. no sign of BBS or rod-cone dystrophy. His father did not have symptoms of BBS.

All experimental procedures were approved by the Ethics Committee at Osaka University (No. 719-2, Osaka, Japan) and conducted in accordance with the Declaration of Helsinki. Written informed consent was obtained from the patient (at the time of the report, a 28-year-old male) and his 61-year-old mother. Both individuals underwent ophthalmologic examinations: BCVA in decimal units, slit-lamp biomicroscopy, fundoscopy, visual field testing with Goldmann perimetry, optical coherence tomography (SSOCT; DRI OCT1, Topcon Corp., Tokyo, Japan), and fundus autofluorescence (Optos, Optos KK, Tokyo, Japan). Genomic DNA was extracted from blood samples using NucleoSpin Blood XL (Macherey-nagel, Düren, Germany). DNA libraries were constructed using SureSelectXT Human All Exon Kit V6 and SureSelectXT Reagent Kit (Agilent, Santa Clara, CA, USA) and then subjected to 100 bp paired-end sequencing on an Illumina HiSeq2500 Platform (Illumina, San Diego, CA, USA). Sequence reads were aligned to the reference human genome (UCSC hg19) in BWA (http://www.bio-bwa.sourceforge.net/) to align short reads after adaptor sequences were removed by Cutadapt (https://cutadapt.readthedocs.io/en/stable/). SAM tools (Version 0.1.17; http://www.samtools.sourceforge.net/) were used for sequence data conversion, sorting, and indexing. To exclude duplicate reads, Picard (http://picard. sourceforge.net) was used. Variants were determined using GATK (http://www.broadinstitute.org/gatk/). ANNOVAR 
Table 1 Primary and secondary signs of BBS in this patient

\begin{tabular}{|c|c|c|c|}
\hline & Age of onset & Clinical information & Intervention \\
\hline \multicolumn{4}{|l|}{ Primary signs } \\
\hline Rod-cone dystrophy & 8 Years old & Visual acuities: 0.01 (right), 0.04 (left), (with mild myopia and astigmatism) & No medication \\
\hline & & $\begin{array}{l}\text { Fundus finding: binocular diffuse retinal degeneration } \\
\text { Visual field: centipede constriction (binocular) } \\
\text { Optical coherence tomography: binocular diffuse thinning of outer retinal layer } \\
(+) \text {, macular atrophy }(+) \text {, macular edema }(-) \text {, cystic changes }(-) \text {, elipsoid } \\
\text { zone }(-) \\
\text { Fundus autofluorescence: binocular mottled pattern }(+) \text {, perifoveal ring }(-)\end{array}$ & \\
\hline Polydactyly & At birth & Both feet & Plastic surgery (19 months old) \\
\hline Obesity & 9 Years old & $\begin{array}{l}\text { Height: } 164 \mathrm{~cm} \\
\text { Weight: } 78.1 \mathrm{~kg} \\
\text { Body mass index (BMI): } 29 \mathrm{~kg} / \mathrm{m}^{2}\end{array}$ & No medication \\
\hline Hypogonadism & & Testosterone: $300-600$ ng/dl & No medication \\
\hline Renal anomalies & 1 Week old & $\begin{array}{l}\text { Cystic kidney } \\
\text { Creatinine: } 1.79 \mathrm{mg} / \mathrm{dl} \\
\text { BUN: } 21 \mathrm{mg} / \mathrm{dl} \\
\text { eGFR cre: } 37.2 \mathrm{~mL} / \mathrm{min} / 1.73 \mathrm{~m}^{2}\end{array}$ & No medication \\
\hline Mental retardation & No & - & - \\
\hline \multicolumn{4}{|l|}{ Secondary signs } \\
\hline Hirschsprung disease & 3 Months old & - & Surgery (28 months old) \\
\hline $\begin{array}{l}\text { Abnormal glucose } \\
\text { tolerance }\end{array}$ & 9 Years old & $\begin{array}{l}\mathrm{HbA} 1 \mathrm{c}: 5.6 \% \text {, } \\
75 \mathrm{~g} \text { oral glucose tolerance test: } 82 \mathrm{mg} / \mathrm{dL} \text { at } 0 \mathrm{~h}, 185 \mathrm{mg} / \mathrm{dL} \text { at } 2 \mathrm{~h}\end{array}$ & No medication \\
\hline Exotropia & NA & - & $\begin{array}{l}\text { Bilateral lateral rectus muscle } \\
\text { recession ( } 14 \text { years old) }\end{array}$ \\
\hline Hypertension & 27 Years old & Blood pressure $=145 / 83 \mathrm{~mm} \mathrm{Hg}$ & $\begin{array}{l}\text { Oral medicine (Azilsartan } 20 \mathrm{mg} \text { and } \\
\text { Amlodipine besilate } 3.47 \mathrm{mg} \text { per day) }\end{array}$ \\
\hline Cataract & NA & Binocular anterior sub-capsular cataract & - \\
\hline Heart diseases & No & - & - \\
\hline Liver fibrosis & No & - & - \\
\hline
\end{tabular}

(http://www.openbioinformatics.org/annovar/) was used to annotate the resulting genetic variants. Rare variants (minor allele frequency $<0.05$ ) were selected using the Exome Sequencing Project, 1000 Genomes Project, and Human Genetic Variation databases; possible pathogenic variants, such as nonsynonymous, nonsense, and frameshift mutations, were extracted from among the retinal degenerative disease-related genes registered in the Ret.Net. ${ }^{\mathrm{TM}}$ database.

Ten candidate pathogenic rare variants in genes related to retinal degenerative diseases were detected in this patient. All were heterozygous variants; however, two novel nonsense (NM_001288781.1 [TTC8_v001]: c.226 C > T, p.Q76X) and frameshift (NM_001288781.1 [TTC8_v001]: c.309_310insTA, p.T103fs) mutations were located in the TTC8 gene (also known as BBS8). Both mutations were validated by direct sequencing of PCR products (Applied Biosystems 3730 DNA Analyzer; Thermo Fisher Scientific K.K., Tokyo, Japan). The primer sets used for PCR were as follows: c.226 C > T, $5^{\prime}$-TGG GTTTTAGGCAGCTTGGA-3' and 5'-ACCATAAGGCA GAACAGAAACCA-3'; c.308_309insAT, 5' -TAGGCCCT GGAACGTCTTTG-3' and 5' - ACCATAAGGCAGAAC AGAAACCA-3'. This mutation is likely to be pathogenic, because the TTC8 gene has been reported as a causative gene for $\mathrm{BBS}^{4}$. The nonsense mutation was located in exon 3 of the TTC 8 gene, thus producing a truncated protein without tetratricopeptide repeats 11 and 15, which are involved in pilus formation and twitching mobility. The frameshift mutation in exon 5 (c.309_310insTA) generates a premature stop codon in exon 6 , which also produces TTC8 lacking normal tetratricopeptide repeats 11 and 15. The premature stop codon is located before the last exon; notably, a mRNA transcribed from a gene with a truncating mutation often undergoes nonsensemediated mRNA decay before translation ${ }^{5}$. Thus, transcripts with nonsense and frameshift mutations are likely to be rapidly degraded to reduce the translation of the truncated TTC8 protein. Therefore, this compound heterozygous patient would not have a functional TTC8 protein to support the formation of the BBSome, leading to the development of BBS. His mother exhibited the heterozygous nonsense mutation, but no frameshift mutation. Although the genetic and clinical data were not available from his father, this patient's BBS was determined to result from a compound heterozygous TTC8 gene mutation.

BBS patients with mutations in the TTC 8 gene comprise only $2.8 \%$ of all BSS patients ${ }^{6,7}$. In Japan, the genetics of four BBS families have been reported: $B B S 2, B B S 5$, and $B B S 7$ homozygotes, as well as a $B B S 10$ compound heterozygote $^{8,9}$. To the best of our knowledge, this is the first BBS patient with a mutation in the TTC8 gene in Japan. Thus far, 16 families with the TTC 8 genetic abnormality 


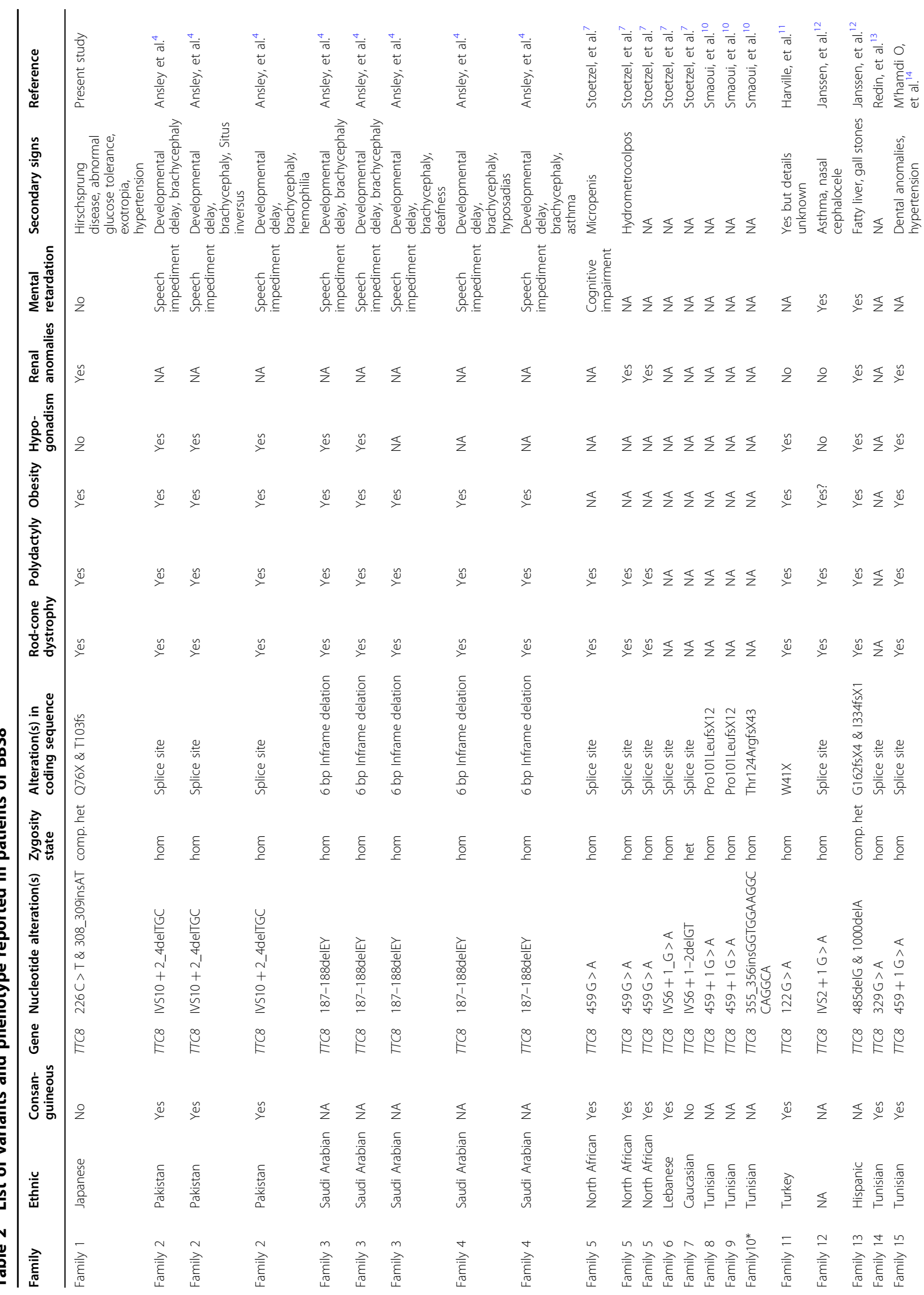




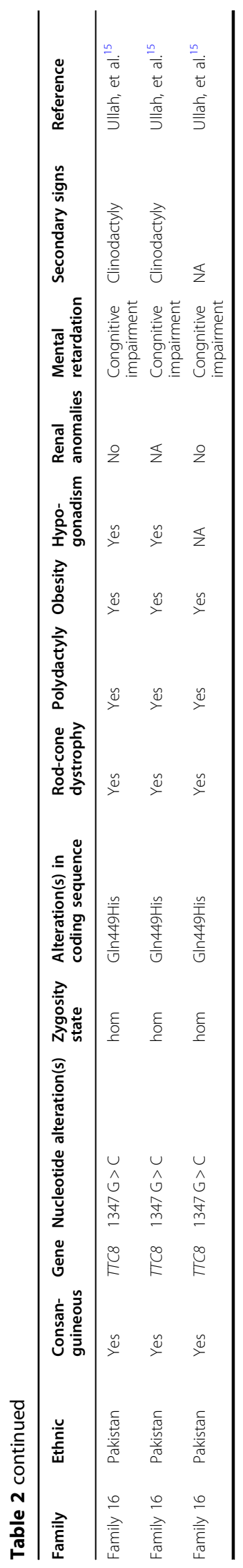

have been reported (Table 2) $)^{4,7,10-15}$. Most of these families have homozygous mutations; only our patient and a Hispanic family were compound heterozygotes. Although full clinical information was not available for some cases, most of the cases in these 16 families exhibit classical BBS without obvious differences in phenotypes.

In summary, we identified a novel compound heterozygous mutation in a Japanese BBS patient by WES. Our findings suggest that WES may be a useful tool for genetic diagnosis and characterization of BBS.

\section{HGV database}

The relevant data from this Data Report are hosted at the Human Genome Variation Database at https://doi.org/10.6084/m9.figshare.hgv.2528; https://doi. org/10.6084/m9.figshare.hgv.2531

\section{Acknowledgements}

We thank E. Suga, M. Morita, Y. Hasegawa, S. Tanaka, and S. Ishino for their technical assistance. We thank Editage (www.editage.jp) for the English language editing. This research was supported by the Project Promoting Clinical Trials for the Development of New Drugs and Medical Devices (Japan Medical Association) from the Japan Agency for Medical Research and Development, AMED.

\section{Author details}

'Department of Ophthalmology, Osaka University Graduate School of Medicine, Osaka, Japan. ${ }^{2}$ Department of Applied Visual Science, Osaka University Graduate School of Medicine, Osaka, Japan. ${ }^{3}$ Department of Medical Innovation, Osaka University Hospital, Osaka, Japan

\section{Conflict of interest}

The authors declare that they have no conflict of interest.

\section{Publisher's note}

Springer Nature remains neutral with regard to jurisdictional claims in published maps and institutional affiliations.

Received: 24 November 2018 Revised: 24 December 2018 Accepted: 10 January 2019.

Published online: 12 March 2019

\section{References}

1. Forsythe, E., Kenny, J., Bacchelli, C. \& Beales, P. L. Managing Bardet-Biedl Syndrome-now and in the future. Front. Pediatr. 6, 23 (2018).

2. Dilan, T. L. et al. Bardet-Biedl syndrome-8 (BBS8) protein is crucial for the development of outer segments in photoreceptor neurons. Hum. Mol. Genet. 15, 283-294 (2018)

3. Beales, P. L., Elcioglu, N., Woolf, A. S., Parker, D. \& Flinter, F. A. New criteria for improved diagnosis of Bardet-Biedl syndrome: results of a population survey. J. Med. Genet. 36, 437-446 (1999).

4. Ansley, S. J. et al. Basal body dysfunction is a likely cause of pleiotropic BardetBiedl syndrome. Nature 425, 628-633 (2003).

5. Hug, N., Longman, D. \& Cáceres, J. F. Mechanism and regulation of the nonsense-mediated decay pathway. Nucleic Acids Res. 44, 1483-1495 (2016).

6. Bin, J. et al. BBS7 and TTC8 (BBS8) mutations play a minor role in the mutational load of Bardet-Biedl syndrome in a multiethnic population. Hum. Mutat. 30, E-737-E746 (2009)

7. Stoetzel, C. et al. BBS8 is rarely mutated in a cohort of 128 Bardet-Biedl syndrome families. J. Hum. Genet. 51, 81-84 (2006).

8. Hirano, M. et al. The first nationwide survey and genetic analysis of BardetBiedl syndrome in Japan. PLOS ONE 10, e0136317 (2015).

9. Kurata, K. et al. Clinical characteristics of a Japanese patient with BardetBiedl syndrome caused by BBS 10 mutations. Jpn J. Ophthalmol. 62 458-466 (2018). 
10. Smaoui, N. et al. Screening of the eight BBS genes in Tunisian families: no evidence of triallelism. Invest. Ophthalmol. Vis. Sci. 47, 3487-3495 (2006).

11. Harville, H. M. et al. Identification of 11 novel mutations in eight BBS genes by high-resolution homozygosity mapping. J. Med. Genet. $\mathbf{4 7}$ 262-267 (2010).

12. Janssen, S. et al. Mutation analysis in Bardet-Biedl syndrome by DNA pooling and massively parallel resequencing in 105 individuals. Hum. Genet. 129, 79-90 (2011).
13. Redin, C. et al. Targeted high-throughput sequencing for diagnosis of genetically heterogeneous diseases: efficient mutation detection in Bardet-Biedl and Alström syndromes. J. Med. Genet. 49, 502-512 (2012).

14. M'hamdi, O. et al. Clinical and genetic characterization of Bardet-Biedl syndrome in Tunisia: defining a strategy for molecular diagnosis. Clin. Genet. $\mathbf{8 5}$ 172-177 (2014).

15. Ullah, A. et al. Sequence variants in four genes underlying Bardet-Biedl syndrome in consanguineous families. Mol. Vis. 23, 482-494 (2017) 